\title{
Importance of tailoring lattice strain in halide perovskite crystals
}

\author{
Hui-Seon Kim (1) and Nam-Gyu Park (10 ${ }^{2}$
}

\begin{abstract}
In this review paper, the residual strain of a polycrystalline halide perovskite film is systematically studied based on its structural inhomogeneity, which is closely correlated to the local carrier dynamics caused by a modulated electronic band structure. Long-range collective strain ordering is responsible for the overall structural properties, consequently determining the optoelectronic properties of the perovskite film. Notably, the perovskite phase stability is strongly affected by the internal strain, favoring a lower energy state. The important parameters affecting the residual strain in a real perovskite film, ranging from thermal stress to lattice mismatch and compositional inhomogeneity, are subsequently introduced along with their impacts on the optoelectronic properties and/or the stability of the crystals.
\end{abstract}

\section{Introduction}

Interest in halide perovskite crystals has exponentially increased since the very first type of solid-state perovskite solar cell (PSC) was released in 2012 1,2 , demonstrating a promising power conversion efficiency (PCE) of approximately $10 \%$ with 500 -hour-long shelf stability ${ }^{1}$. Tremendous efforts have been made to raise the performance of PSCs, and the state-of-the-art technology in PSCs drove its PCE into a remarkable value, $25.5 \%$ in $2020^{3}$, making PSCs highly competitive with conventional photovoltaics and one of the most promising next-generation solar cells ${ }^{4-6}$. Most of the dominant progress in PSCs was achieved through the compositional and morphological engineering of halide perovskite crystals via a macroscopic approach ${ }^{7}$. The composition of perovskite crystals, initially governed by a single cation- and halide-based methylammonium lead iodide $\left(\mathrm{MAPbI}_{3}\right)^{1,2}$, evolved into a cutting-edge mixed cation- and halide-based system where formamidinium cation $\left(\mathrm{FA}^{+}\right)$and iodide $\left(\mathrm{I}^{-}\right)$assume a majority in

\footnotetext{
Correspondence: Hui-Seon Kim (hui-seon.kim@inha.ac.kr) or

Nam-Gyu Park (npark@skku.edu)

'Department of Chemistry and Chemical Engineering, Inha University, Incheon 22212, Korea

${ }^{2}$ School of Chemical Engineering, Sungkyunkwan University, Suwon 16419 , Korea
}

composition along with cesium cation $\left(\mathrm{Cs}^{+}\right), \mathrm{MA}^{+}$and bromide $\left(\mathrm{Br}^{-}\right)^{8-11}$. Furthermore, the morphology of the perovskite was set as the bulk film ${ }^{12,13}$, complementarily guided by fundamental studies revealing extremely outstanding optoelectronic properties of halide perovskites $^{14-17}$. Though the PCE of PSCs has been significantly raised by the aid of recent evolution with $2 \mathrm{D} /$ $3 \mathrm{D}$ hybrid perovskite architectures ${ }^{18-20}$, there is still room for improvement. Sophisticated micro tuning of the lattice strain of the perovskite film deserves attention for PSCs, aiming at a further boost to close to the theoretical limit as well as ensured intrinsic phase stability.

Strain engineering is a very useful strategy in crystals to minutely modulate electronic properties by tuning the band structure. Strain tailoring is frequently performed on various crystals, such as germanium ${ }^{21}, \mathrm{WSe}_{2}{ }^{22}$, and $\mathrm{GaAs}^{23}$, to manipulate their optoelectronic properties. Halide perovskite also provides substantial room for the strain to be involved as a key parameter to alter its electronic band and thus affect the resulting properties. The halide perovskite film shows a residual strain caused by inhomogeneity mainly due to its polycrystalline nature $^{24,25}$. Any strain in the lattice accordingly has an impact on the electronic band structure by interfering with the bonding strength of the $\mathrm{PbI}_{6}$-coner sharing 
octahedral frame ${ }^{26,27}$. Importantly, strain tuning can play a key role in determining the intrinsic stability of the photoactive perovskite phase from a thermodynamic point of view ${ }^{28}$. Therefore, strain engineering would be a demanding approach for current PSCs toward the next breakthrough. In this review paper, the effect of lattice strain on the relevant optoelectronic properties of halide perovskites is discussed from the electronic band structure to the carrier dynamics and the crystal phase stability as well. Subsequently, methods to perform strain engineering are proposed for halide perovskites by addressing the affecting parameters. Finally, the effective scope of the strain-induced benefits and the expected limits are examined from a practical point of view to provide an overall perspective.

\section{Effect of the lattice strain on optoelectronic properties}

The halide perovskite in PSCs shows polycrystallinity due to its film growth at low temperature based on the solution process ${ }^{29}$. Therefore, the inhomogeneity is necessarily inherent in the perovskite film, where distinctive electronic properties are observed even in the same grain ${ }^{30}$, which is indicative of the underlying lattice mismatch. The strain induced by local lattice mismatch tends to be relieved by a relaxation mechanism. As the thickness of the perovskite film increases, the increased elastic energy helps the perovskite film dissipate the induced $\operatorname{strain}^{31}$. On the other hand, structural dislocation may occur by generating defects to relieve the strain when the film thickness is thinner than the threshold for elastic relaxation ${ }^{31}$. The effect of elastic relaxation across the film is negligible in real devices when considering that the threshold thickness of the halide perovskite is far beyond $3 \mu \mathrm{m}^{32}$, compared to the ca. $500 \mathrm{~nm}$ thick perovskite film in PSCs. Accordingly, the polycrystalline perovskite film is likely to exhibit residual strain and to be involved in bonding hybridization ${ }^{33}$. The electronic properties of the polycrystalline halide perovskite film are highly subject to macroscopic internal strain, which is determined by the overall localized residual strains on the microscale ${ }^{33-35}$.

\section{Electronic band structure}

Perovskite crystals in the form of the bulk film can be exposed to relatively in-plane or out-of-plane strains, indicating strains parallel or perpendicular to the substrate, respectively. In halide lead perovskites, the valence band edge is governed by the antibonding between $\mathrm{Pb}-6 \mathrm{~s}$ and halide (X)-p orbitals, while the conduction band edge is mostly determined by nonbonding localized $\mathrm{Pb}-6 \mathrm{p}$ orbitals $^{26,27}$. The strain-induced lattice expansion/contraction modulates the bond strength in the lattice, leading to a change in the electronic band structure, particularly in the valence band edge. Figure $1 \mathrm{a}-\mathrm{c}$ shows schematic illustrations of the lattice structure bearing concentrated in-plane strain, implying tensile strain at the bottom surface in Fig. 1a, a strain-free state in Fig. 1b, and compressive strain at the bottom surface in Fig. 1c. Grazing incidence X-ray diffraction (GIXRD) is a wellestablished tool to characterize the residual strain existing within a material ${ }^{36,37}$. GIXRD adopts a small incidence angle of $\psi$ over a fixed $2 \theta$ corresponding to $(h k l)$ and detects the resulting diffraction intensity from different orientations of the scattering vector $\left(\mathrm{N}_{\mathrm{k}}\right)^{35}$, which is not parallel to the substrate normal, as illustrated in Fig. 1d. The GIXRD patterns in Fig. 1e-g effectively reflect the inplane lattice change by tilting a substrate-loaded instrument with an angle, $\psi$. In other words, the X-ray diffraction (XRD) peak at $\psi=0$ (no tilting) is directly determined by the distance between lattice planes parallel to the substrate, which thus involve no information about the in-plane lattice expansion or contraction. As the tilting angle $\psi$ increases, however, the in-plane lattice distance starts contributing to the beam path and consequently affects the XRD peak position. The residual strain, $\varepsilon(h k l)$, can therefore be determined by the difference in the interplanar spacing of $d$ between $\mathrm{N}_{\mathrm{k}}$ and $\mathrm{N}_{0}$, $\varepsilon(h k l)=\left(d_{\psi}-d_{0}\right) / d_{0}$. The tensile strain-induced film shows a gradual peak shift toward a lower $2 \theta$ as $\psi$ increases (Fig. 1e), implying lattice expansion, whereas the strain-free film shows constant peak positions regardless of $\psi$ (Fig. 1f). A peak shift to a higher $2 \theta$ is observed with increasing $\psi$ in Fig. 1g, which is in accordance with the expected lattice contraction by the compressive strain. Under tensile strain, lattice expansion occurs, resulting in a downshift of the valence band edge as a consequence of weakened antibonding ${ }^{26,27}$. On the other hand, the lattice contraction by compressive strain strengthens the overlap of the antibonding and thus lifts the valence band edge up compared to the strain-free film.

The tendency is well described in Fig. 2, showing the effect of strains on the calculated band structure (Fig. 2a) and the estimated band gap $\left(V_{\mathrm{g}}\right)$ by experimental results (Fig. 2c). A narrower band gap $\left(V_{\mathrm{g}}\right)$ is observed from the compressive strain compared to the case of the strain-free film. On the other hand, the tensile strain induces an opposite effect on the band structures, leading to a wider $V_{\mathrm{g}}$ with increasing strain (Fig. 2b). As expected, the effect of strain on the valence band edge is more pronounced than that of the conduction band edge ${ }^{26,27}$.

Furthermore, the observed difference in the band energy level and $V_{\mathrm{g}}$ by varying the strain is dependent on the composition of the perovskite films. Nevertheless, it is found that a strain within $\pm 2.5 \%$ (negative value for the compressive strain and positive value for the tensile strain) has been generally used as a feasible strain window in various studies, providing a controllable margin of $V_{\mathrm{g}}$ within $50 \mathrm{meV}^{25,32,35,38}$. Notably, the beneficial effect is 


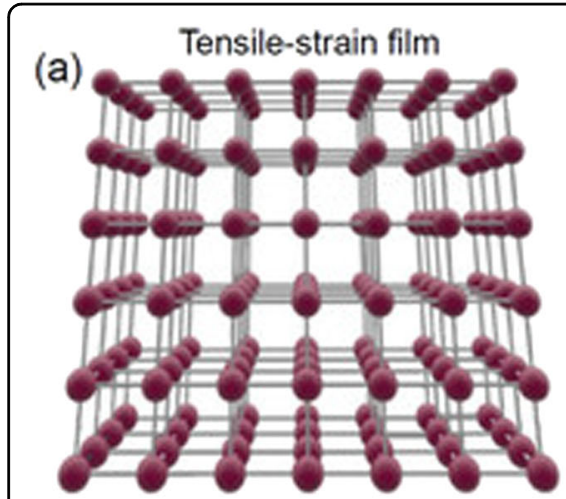

(b) Strain-free film

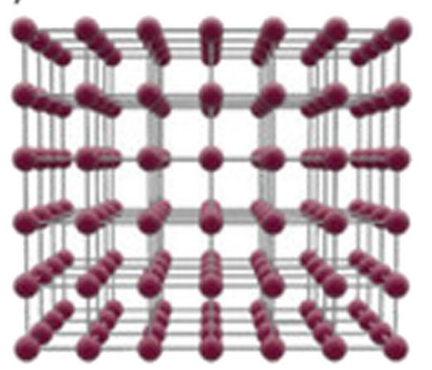

(c)

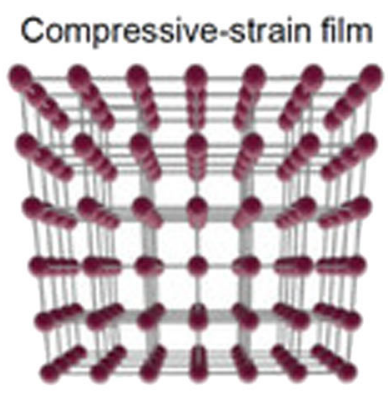

(d)
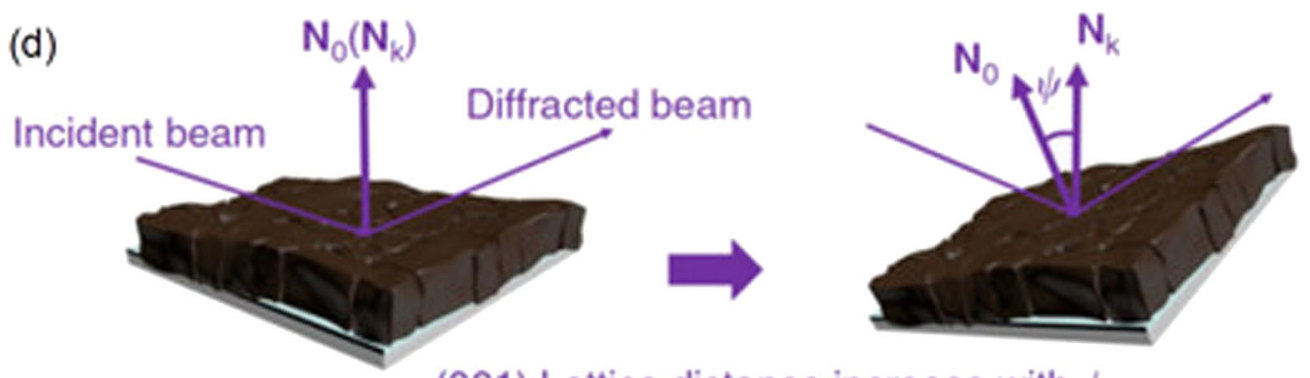

(001) Lattice distance increase with $\psi$
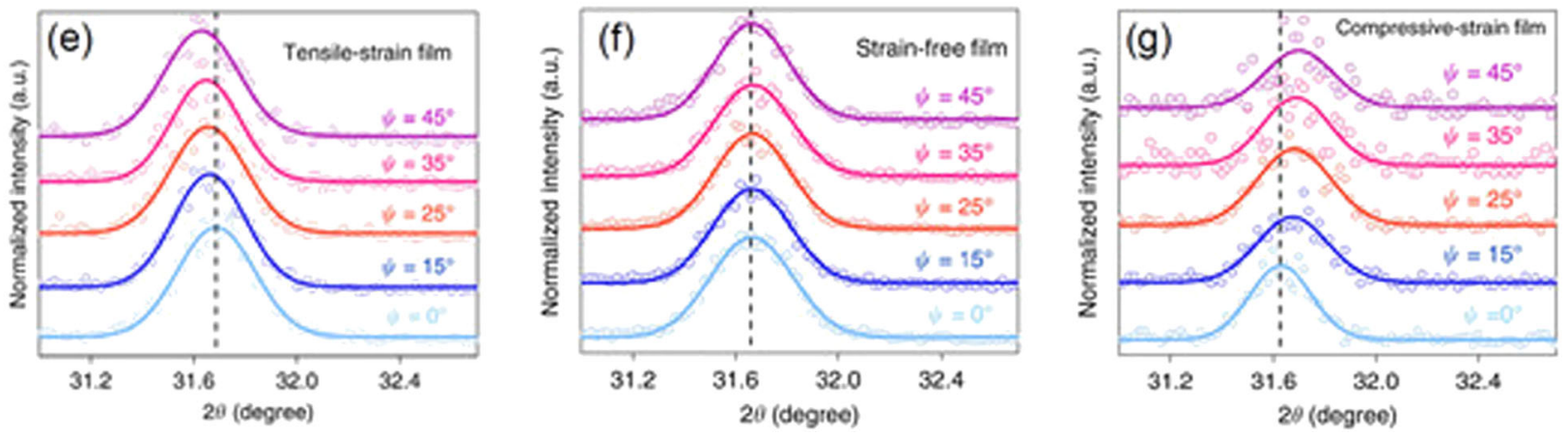

Fig. 1 Schematic illustration of in-plane residual strain and its effect on the GIXRD patterns. Schematic illustration of the lattice structure (a) with tensile strain, (b) without strain, and (c) with compressive strain at the bottom surface. $\mathbf{d}$ Schematic representation of the residual strain measurement in the XRD system. The in-plane residual strain can be observed by adjusting the instrument tilt angle $\psi$ with a fixed crystal plane. $N_{0}$ indicates the sample normal direction, while $N_{k}$ is the diffraction vector. GIXRD spectra with increasing tilt angles $(\psi)$ of the $(\mathbf{e})$ tensile-strained, (f) strain-free, and (g) compressive-strained films at a depth of 50nm from the top surface. Reprinted with permission from Ref. ${ }^{35}$. Copyright 2019 Nature Publishing Group.

mostly observed when the inherent strain of the perovskite film ranges from a slight compressive strain $(-1.5 \%)$ to a strain-free condition $(0 \%)^{25,32,35}$.

The effect of spin-orbit coupling (SOC) on the electronic band structure can be reversed when a phase transition is accompanied by intense strain, particularly under high pressure in the GPa range ${ }^{39-41}$. The halide perovskite crystals close to the cubic phase and pseudocubic phase represent $\mathrm{Pb}-\mathrm{X}-\mathrm{Pb}$ with a bond angle of $180^{\circ}$ as in the $\alpha-$ phase lattice. Therefore, the tensile strain effectively weakens the orbital coupling between $\mathrm{Pb}-6 \mathrm{~s}$ and $\mathrm{X}-\mathrm{p}$, while the compressive strain readily strengthens the orbital overlap, as discussed earlier. On the other hand, the intense strain can cause a phase transition from the cubic $(\alpha)$ to the orthorhombic $(\delta)$ phase. A stark distortion of the corner-sharing octahedral structure of $\mathrm{PbI}_{6}$ occurs under the $\delta$-phase due to the Jahn-Teller effect ${ }^{40}$. The distortion makes the $\mathrm{Pb}-\mathrm{X}$ $\mathrm{Pb}$ bond angle deviate from $180^{\circ}$, where the induced strain exerts more influence on the bond angle than on the bond distance. Consequently, the compressive strain reduces the $\mathrm{Pb}-\mathrm{X}-\mathrm{Pb}$ bond angle, leading to a blueshift in the electronic bandgap based on the reduced $\mathrm{SOC}^{39-41}$. 

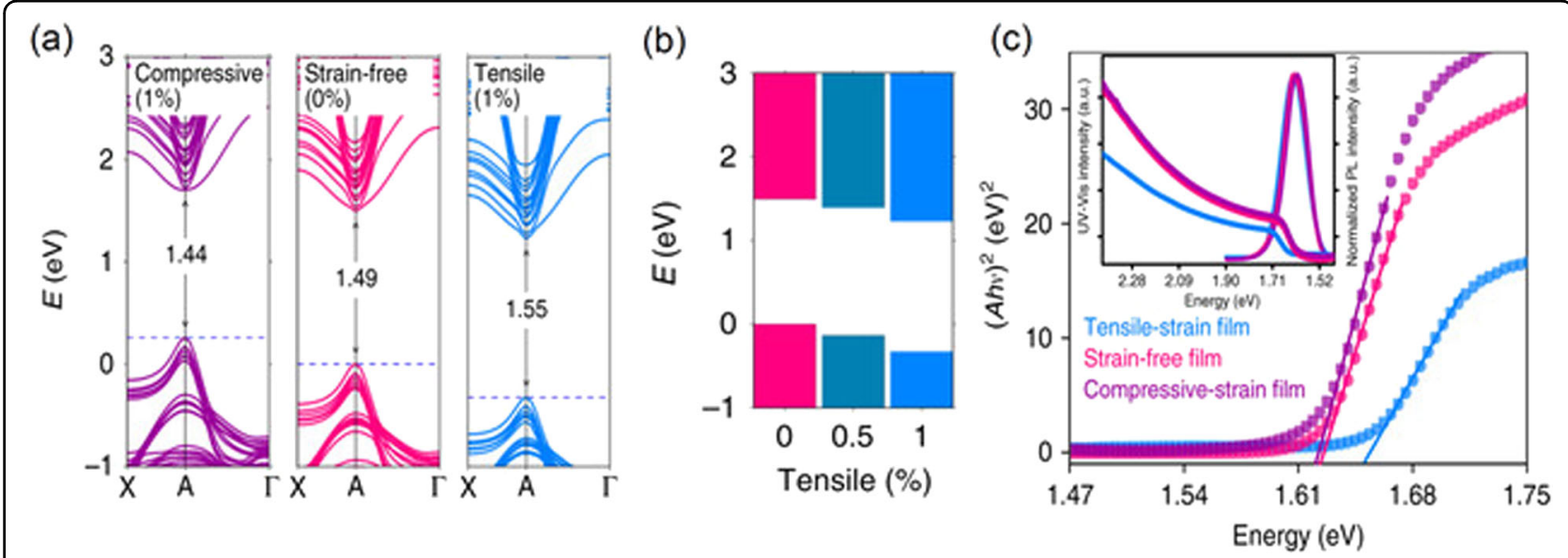

Fig. 2 Strain-induced electronic structure analysis. a Calculated band structures with compressive (-1\%), strain-free (0\%), and tensile (1\%) strains from first-principle density functional theory (DFT)-based approaches. The vacuum energy level is used as a reference for the band alignment. b Estimated band-edge energies as the tensile strain in perovskite films increases. c Tauc plots of the perovskite film under tensile strain (blue), strainfree (pink), and compressive strain (purple). Ultraviolet-visible absorption spectra for the Tauc plots by Kubelka-Munk transformation and PL spectra are shown as inset figures. Reprinted with permission from Ref. ${ }^{35}$. Copyright 2019 Nature Publishing Group.

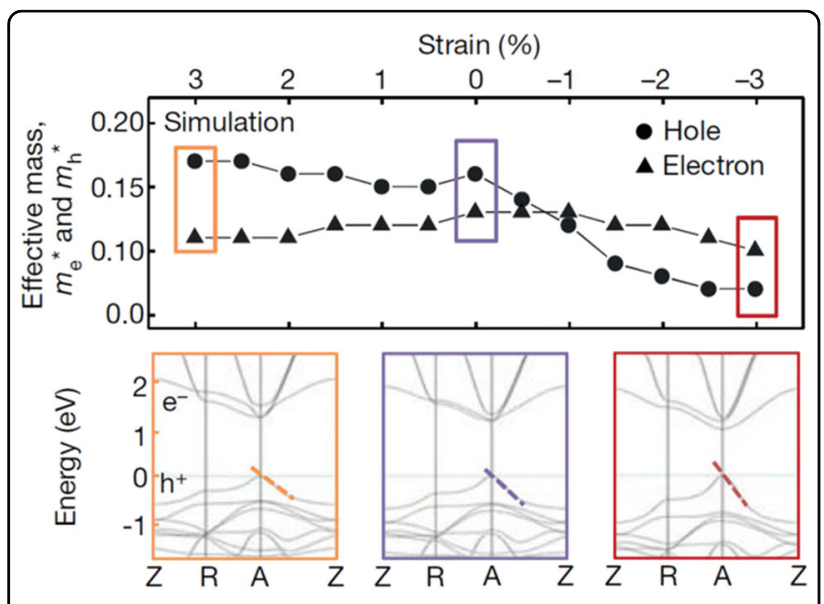

Fig. 3 Calculated electron effective mass $\left(m_{\mathrm{e}}^{*}\right)$ and hole effective mass $\left(m_{\mathrm{h}}{ }^{*}\right)$ as a function of strain level (top panel). Simulated electronic band structures (bottom panel) under three conditions, 3\% (yellow), $0 \%$ (purple) and $-3 \%$ (red), where the dashed lines represent the dispersivity of the valence band. The $Z, R$, and A points correspond to high-symmetry points in the first Brillouin zone of the tetragonal lattice. Reprinted with permission from Ref. ${ }^{32}$. Copyright 2020 Nature Publishing Group.

\section{Carrier dynamics}

Strain-induced lattice deformation can modulate the electronic band structure and thus consequently affect the carrier dynamics. Figure 3 simulates the effective mass $\left(m^{*}\right)$ as a function of strain with corresponding band curvature from first-principle calculation of $\alpha-\mathrm{FAPbI}_{3}{ }^{32}$, taking majority components in the most recent mixedperovskite systems ${ }^{8-11,18-20}$. The E-k dispersion of the valence band of $\alpha-\mathrm{FAPbI}_{3}$ varies with changing strain, showing a more dispersed configuration for the tensile strain and less curvature for the compressive strain. Therefore, a reduced hole effective mass $\left(m_{\mathrm{h}}{ }^{*}\right)$ is expected from the compressive strain, implying a better holetransfer characteristic in the perovskite layer. The electron effective mass $\left(m_{\mathrm{e}}{ }^{*}\right)$ is comparably constant based on the small curvature change observed from the conduction band structure. It is notable that the strain exerts an effect on the overall carrier dynamics mostly by altering the hole-related properties.

The hole mobility is increased under compressive strain owing to the reduced $m_{\mathrm{h}}{ }^{*}$ until the intense strain causes considerable dislocations ${ }^{16}$. A compromise between the lower $m_{\mathrm{h}}{ }^{*}$ and the higher trap density due to the compressive strain leads to the highest hole mobility at $-1.2 \%$ strain for $\alpha-\mathrm{FAPbI}_{3}$ (Fig. 4a) ${ }^{32}$. The hole mobility tendency is well in accordance with the transient photocurrent results, showing the best performance at $-1.2 \%$ strain for $\alpha-\mathrm{FAPbI}_{3}$ (Fig. 4b) ${ }^{32}$. The carrier dynamics observed from $\left(\mathrm{FAPbI}_{3}\right)_{0.85}\left(\mathrm{MAPbBr}_{3}\right)_{0.15}$, one of the most widely used mixed-perovskite compositions, also correspond to the strain effect shown in $\alpha-\mathrm{FAPbI}_{3}$. The $\left(\mathrm{FAPbI}_{3}\right)_{0.85}\left(\mathrm{MAPbBr}_{3}\right)_{0.15}$ under tensile strain demonstrates lower hole mobility than the strain-free film (Fig. 4c), as expected from the increased $m_{h}{ }^{*}$ of $\alpha-\mathrm{FAPbI}_{3}$ with tensile strain. The substantial difference in the measured hole mobility between $\alpha-\mathrm{FAPbI}_{3}$ and (FAP$\left.\mathrm{bI}_{3}\right)_{0.85}\left(\mathrm{MAPbBr}_{3}\right)_{0.15}$, even after considering the compositional difference, may arise from the different film qualities and more significantly from the different analysis methods ${ }^{42}$. Nonetheless, the observed tendencies of the strain-induced hole mobility are well in line with each other. In Fig. 4d, the transient photocurrent of $\left(\mathrm{FAPbI}_{3}\right)_{0.85}\left(\mathrm{MAPbBr}_{3}\right)_{0.15}$ under tensile strain shows a 

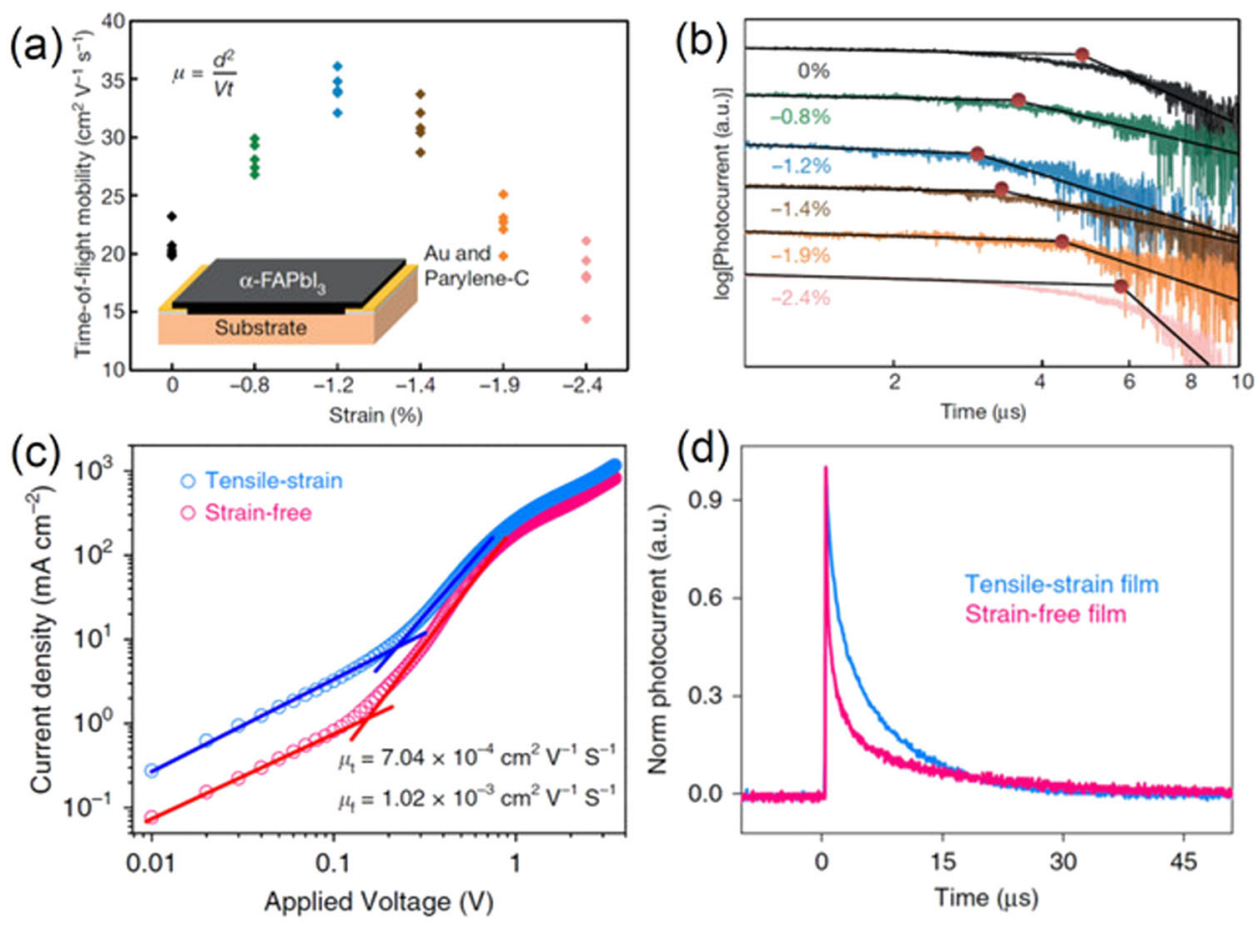

Fig. 4 Effect of strain on the carrier mobility and extraction. a Carrier mobility-strain plots where the time-off light carrier mobility $(\mu)$ is based on the inset equation ( $d=$ sample thickness, $V=$ applied voltage, and $t=$ carrier transit time). The sample used for mobility measurement is shown in the inset figure. Five samples are used at each strain level. Colored symbols are matched with the strain level indicated in (b). b Transient photocurrent decay of a-FAPbl 3 under different compressive strain levels. The inflection point, indicated by a solid red circle, reflects the switching moment of the charge transporting carriers from the majority to the minority carriers. c Current-voltage curves including the space-charge-limited current (SCLC) region, measured from a hole-only device with (blue) and without (pink) tensile strain. $\mathbf{d}$ Transient photocurrent decay of PSCs with blue and without (pink) tensile strain. Reprinted with permission from Ref. ${ }^{32}$. Copyright 2020 Nature Publishing Group and Ref. ${ }^{35}$. Copyright 2019 Nature Publishing Group.

$12.96 \mu$ s decay time, while it is highly reduced to $1 \mu \mathrm{s}$ in the strain-free film ${ }^{35}$, indicating that the tensile strain effectively impedes hole extraction. Similarly, timeresolved photoluminescence (TR-PL) results demonstrate retarded hole extraction at the interface of (FAP$\left.\mathrm{bI}_{3}\right)_{0.85}\left(\mathrm{MAPbBr}_{3}\right)_{0.15}$ with a longer quenching time under tensile strain ${ }^{35}$.

The enhanced charge extraction is readily reflected in the improved photovoltaic parameters, especially in the fill factor $(\mathrm{FF})$ and open-circuit voltage $\left(V_{\mathrm{OC}}\right)$. The ideality factor, $n$, is estimated by the slope of the $V_{\mathrm{OC}}$-light intensity curve to evaluate the recombination behavior, where $n$ approaches 2 as trap-assisted recombination prevails over ideal radiative recombination $(n \approx 1)^{43} \cdot n$ is decreased from 1.55 to 1.05 when the tensile strain is removed, which is responsible for the improved FF and $V_{\mathrm{OC}}$ in the device with the tensile strain-alleviated perovskite film ${ }^{35}$. It is noted that the component variation in each perovskite film and its singularity in the experimental process should be considered with care for interpretation of the results. The compressive strain in the $\mathrm{MAPbBr}_{3}$ film leads to an increased carrier lifetime with reduced trap density ${ }^{38}$, in accordance with the above interpretation. In contrast, the $\mathrm{MAPbI}_{3}$ film and the triple cation-based film $\left(\mathrm{MA}_{0.15} \mathrm{FA}_{0.79} \mathrm{Cs}_{0.06} \mathrm{~Pb}\left(\mathrm{I}_{0.85} \mathrm{Br}_{0.15}\right)_{3}\right)$ show a decreased PL intensity under compressive residual strain due to the predominant trap density ${ }^{30}$. The strain effect from the perspective of defect generation will be intensively discussed in the following section ("Crystallinity") as closely related to nonradiative recombination.

\section{Crystallinity}

The local strain is naturally expected from the polycrystalline perovskite film, where the strain-induced structural defects readily govern the nonradiative recombination ${ }^{44,45}$. The local structural properties on the microscale can be studied by using micro-XRD ( $\mu$ XRD). Figure 5 shows the spatial maps of the peak scattering vector, $q$, of the azimuthally integrated $<220\rangle$ planes (Fig. 5a) and $<222>$ plane (Fig. 5b) of the $\mathrm{MAPbI}_{3}$ film with a grain size of $0.5 \sim 1 \mu \mathrm{m}$. The $q$ mapping apparently confirms the local structural heterogeneity in the $\mathrm{MAPbI}_{3}$ film. The two horizontal slices (indicated as $\mathbf{d}$ and $\mathbf{e}$ ) taken from <220> show subtle shifts in the peak position accompanied by irregular peak broadening, indicating a microscale structural inhomogeneity of $\sim 0.15 \%$ in the 

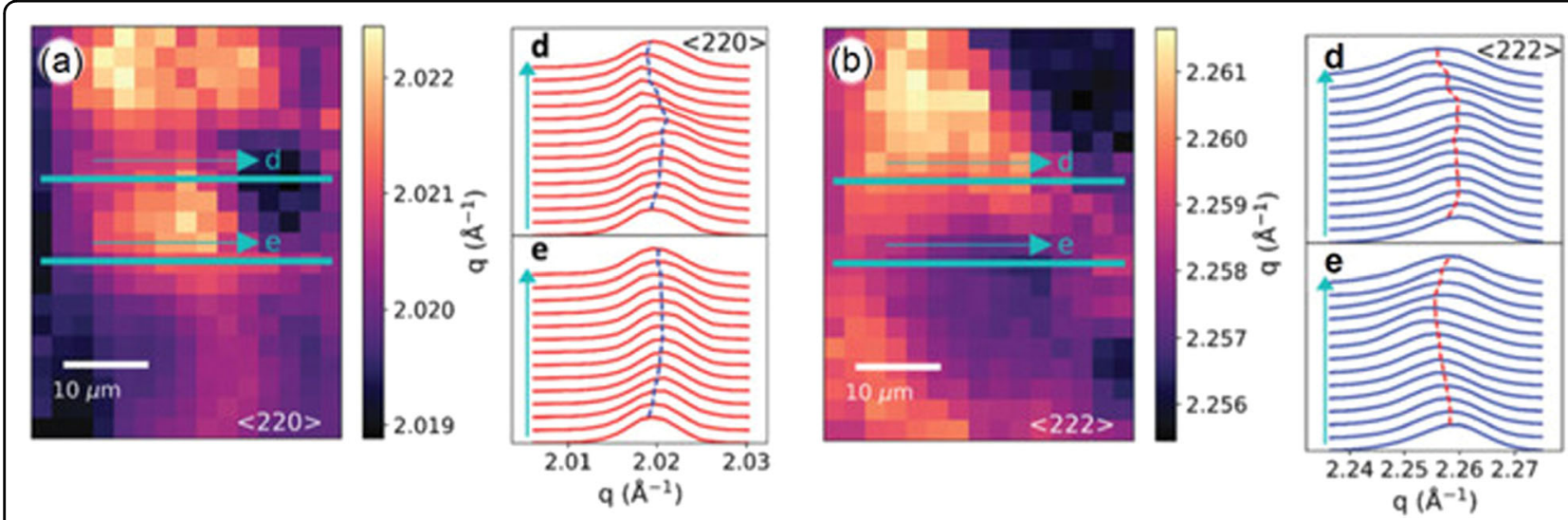

Fig. 5 Local $\mathbf{q}$ vector for $<\mathbf{2 2 0}>$ and $<\mathbf{2 2 2}>$ of $\mathbf{M A P b l}_{\mathbf{3}}$ film. Local q maps from an MAPbl film, indicating (a) $<220>$ and (b) $<222>$ diffraction peaks from $\mu X R D$. $\mathbf{d}$, e Diffraction patterns along the line (indicated with green for $\langle 220\rangle$ (red) in (a) and for $\langle 222\rangle$ (blue) in (b)) chosen from q maps. Vertical dashed lines in $(\mathbf{d}, \mathbf{e})$ represent peak positions. Reprinted with permission from Ref. ${ }^{30}$. Copyright 2019 Royal Society of Chemistry.
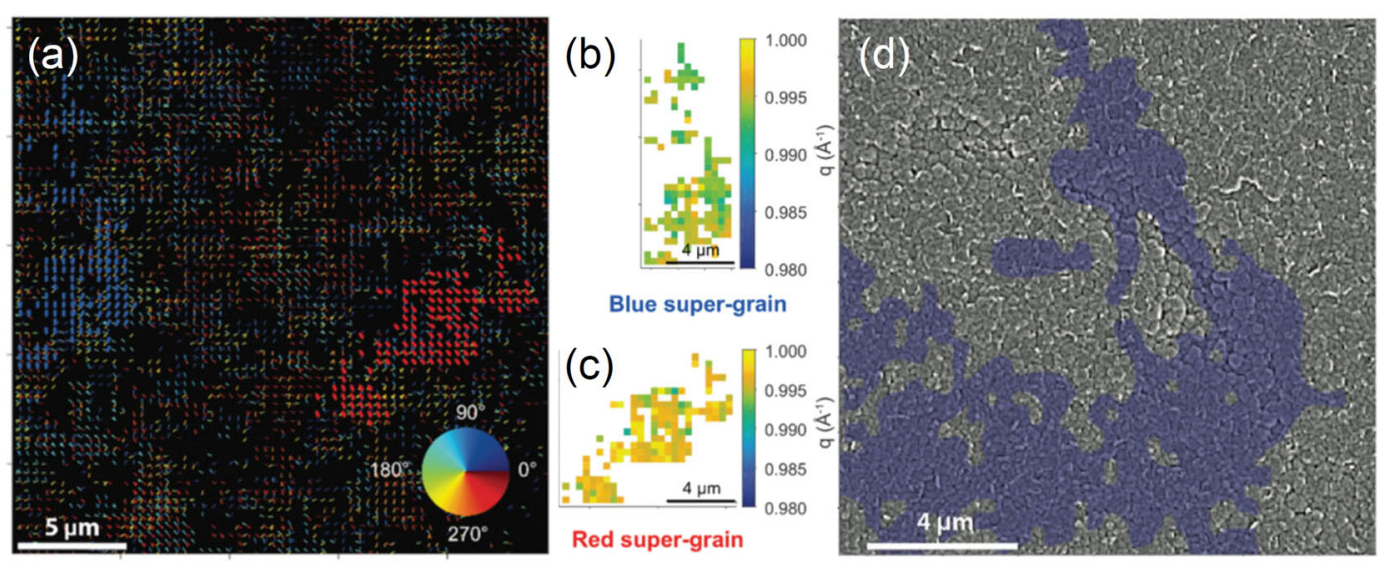

Fig. 6 Similarity in strain over supergrains. a Quiver plot from $\mathrm{nXRD}$ of $\mathrm{MAPb}_{3}$, where highlighted two largest regions indicate supergrains with $<110>$ reflection. The scattering vector $q$ maps corresponding to the $(\mathbf{b})$ blue and (c) red supergrains shown in the quiver plot. $\mathbf{d}$ Overlay image of the $<210>$ supergrains (purple) from the quiver plot of $\mathrm{MA}_{0.15} \mathrm{FA}_{0.79} \mathrm{Cs}_{0.06} \mathrm{~Pb}\left(\mathrm{I}_{0.85} \mathrm{Br}_{0.15}\right)_{3}$ and the corresponding SEM image. Reprinted with permission from Ref. ${ }^{30}$. Copyright 2019 Royal Society of Chemistry.

local $q$ vector for $<220>$. The $\mu$ XRD patterns from the two horizontal lines exhibit parallel coupling over a long range $(\sim 10 \mu \mathrm{m})$, while the two lines from $<222>$ represent antiparallel coupling, which suggests the presence of a long-range region across several grains, sharing similar structural properties across subtle $q$ variations.

The locally oriented strain feature over the long range is more specifically studied by performing nanofocus XRD (nXRD) on the nanoscale. A quiver plot of the <110> orientation of the $\mathrm{MAPbI}_{3}$ film is shown in Fig. 6a, where the diffraction information is represented as a colored arrow in the spatial position. The blue and red parts in the quiver plot indicate supergrains sharing similar crystallographic properties. Figure $6 \mathrm{~b}, \mathrm{c}$, corresponding to the blue and red supergrains, respectively, reveal a similar strain feature over the large supergrains, though a subtle strain inhomogeneity still exists, as observed from the $\mu \mathrm{XRD}$ results. Remarkably, the largest supergrain for $<210>$ of the $\mathrm{MA}_{0.15} \mathrm{FA}_{0.79} \mathrm{Cs}_{0.06} \mathrm{~Pb}\left(\mathrm{I}_{0.85} \mathrm{Br}_{0.15}\right)_{3}$ film is as large as $\sim 25 \mu \mathrm{m}^{2}$, far beyond a single grain size of $\sim 1 \mu \mathrm{m}^{2}$, overlaid with the SEM image in Fig. 6d. Consequently, the supergrain concept based on a similarity in crystallinity is more important than other concepts in determining the local collective strain boundaries to evaluate structural properties.

The residual strain in the crystal is responsible for defect formation. Confocal PL measurements in the same $\mu \mathrm{XRD}$ region help directly correlate the local strain with the local radiative recombination behavior. Figure 7(a) represents the (compressive) strain mapping estimated from the $\mu \mathrm{XRD}$ map of the $\mathrm{MAPbI}_{3}$ film, where the indicated correlation region in the map matches the 


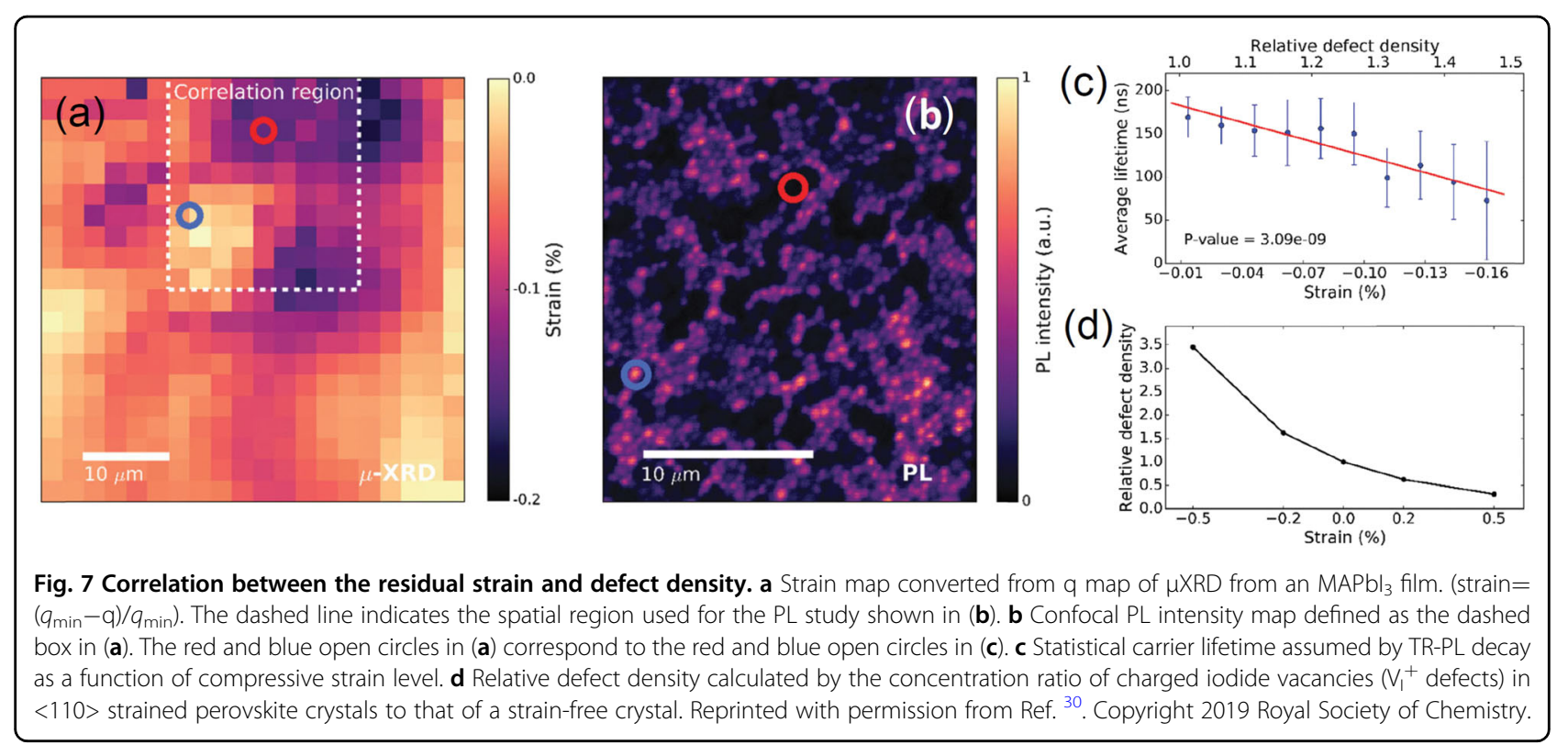

confocal PL mapping in Fig. 7b. Importantly, the monitored local PL heterogeneity is subjected to local strain distributions over the map. The comparably high compressive strain results in a low PL intensity (red open circle), while a high PL intensity is measured at the local point with negligible strain (blue open circle). The correlation between the carrier lifetime from TR-PL and the local strain is plotted in Fig. 7c, where the PL lifetime is seen to gradually decrease as the compressive strain is increased, suggesting structural defects with the compressive strain. Figure $7 \mathrm{~d}$ depicts the local defect concentration as a function of the compressive $<110\rangle$ strain by using a first-principle atomic model. It is assumed that the charged iodide vacancy $\left(\mathrm{V}_{\mathrm{I}}^{+}\right)$concentration is increased by applying compressive strain $^{30}$, indicative of pronounced nonradiative recombination assisted by generated defects under the high compressive strain level in the crystal.

It is worth noting that the collective structural/strain properties should be considered to correlate with the bulk properties over the local crystalline heterogeneity with a subtle variation, as discussed earlier. A peak broadening in $\mathrm{PL}$ and XRD is usually expected with residual strain owing to a reduced crystallinity on the macroscale. The $\alpha$ $\mathrm{FAPbI}_{3}$ film under compressive strain results in PL peak broadening $^{32}$, which is attributed to the reduced crystallinity coupled with the enhanced carrier-phonon coupling, as confirmed by temperature-dependent PL studies $^{32}$. Similarly, peak broadening in high-resolution $\mathrm{XRD}$ patterns is also observed for $\alpha-\mathrm{FAPbI}_{3}$ with increasing compressive strain and with reducing film thickness $^{32}$. When a film is thinner than a critical thickness for plastic relaxation, the compressive strain level is directly reflected in the reduced crystallinity. After passing through the critical thickness, however, plastic relaxation occurs to relieve the compressive strain by generating dislocations ${ }^{16}$. Therefore, the increased PL intensity and the increased $V_{\mathrm{g}}$ are observed from a thick film whose thickness allows the compressive strain to be relieved by dislocations ${ }^{32}$. In the meantime, the increased trap density results from the increased compressive strain level as a consequence of defect propagation into the $\alpha-\mathrm{FAPbI}_{3}$ film by plastic dislocations ${ }^{32}$, which is in accordance with the microscale defect tendency under compressive strain for the $\mathrm{MAPbI}_{3}$ film.

\section{Crystal phase stability}

One of the most important merits in tailoring lattice strain lies in ensuring the phase stability of the metastable perovskite film. The composition of recent perovskites has converged toward $\alpha-\mathrm{FAPbI}_{3}$ as a major component in mixed-perovskite crystals ${ }^{8-11,18-20}$. The $\alpha-\mathrm{FAPbI}_{3}$ phase shows a spontaneous transition to the nonperovskite $\delta$ phase (photoinactive yellow phase) at room temperature ${ }^{46}$. The instability of the $\alpha-\mathrm{FAPbI}_{3}$ phase, therefore, promotes the incorporation of foreign ions (e.g., $\mathrm{MA}^{+}$, $\mathrm{Cs}^{+}, \mathrm{Br}^{-}$) to inhibit the transition to the $\delta$ phase by establishing an energetically stable $\alpha$ phase of the perovskite crystals with mixed components ${ }^{47}$. Notably, the residual strain in the lattice significantly influences the phase stability of $\alpha-\mathrm{FAPbI}_{3}$, leading to superb phase stability under compressive strain even in the absence of foreign ions ${ }^{32}$. Two $\alpha-\mathrm{FAPbI}_{3}$ films with the internal compressive strain (pink lines) and without strain (black lines) are prepared, and their long-term stabilities are monitored by XRD (Fig. 8a), PL (Fig. 8b), and Raman 

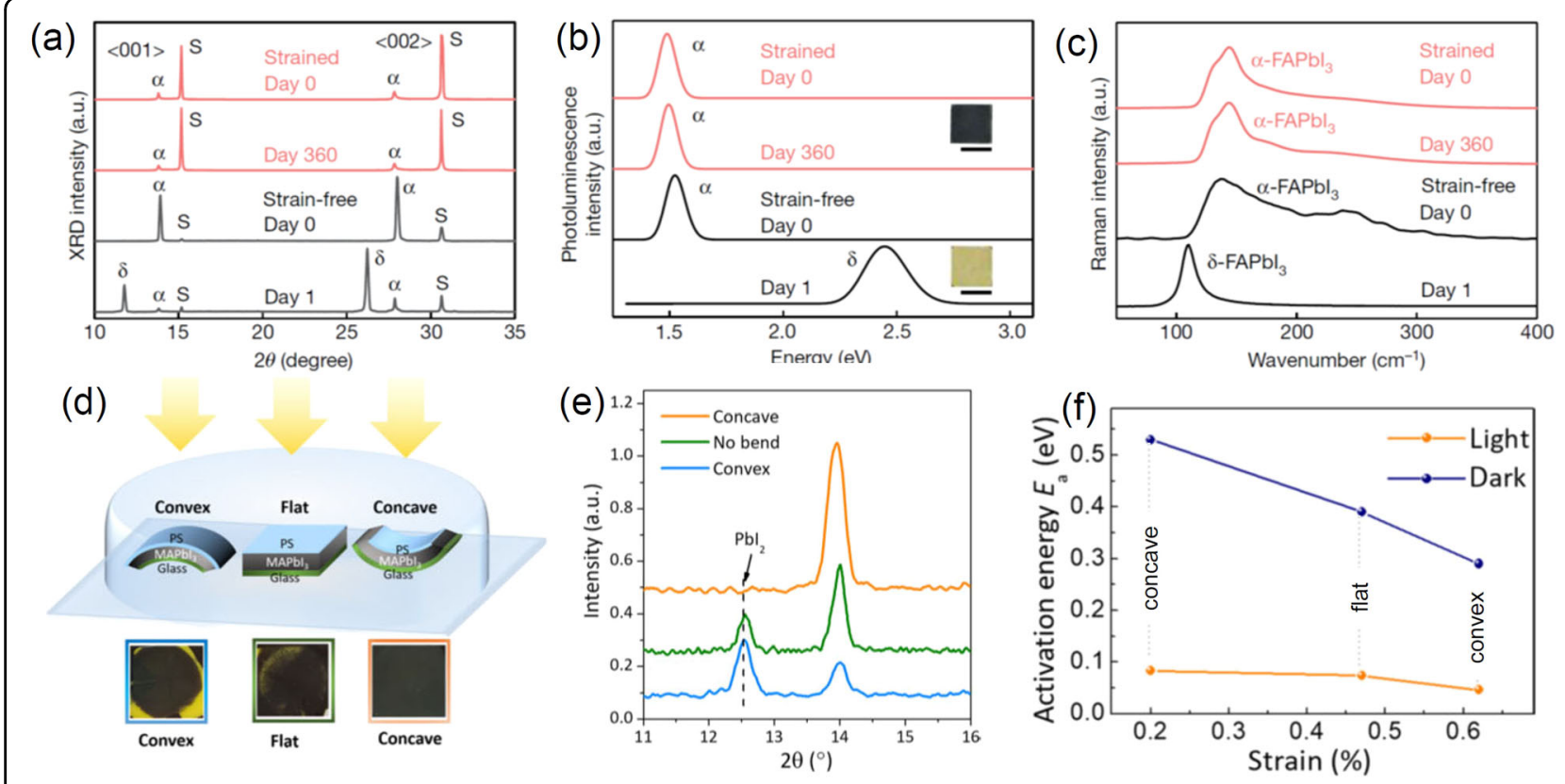

Fig. 8 Effect of in-plane strain on the crystal phase stability of a- $\mathrm{FAPbl}_{3}$ and $\mathrm{MAPbl}_{3}$. (a) XRD patterns, (b) PL spectra, and (c) Raman spectra of $\mathrm{a}-\mathrm{FAPbl}_{3}$ on $\mathrm{MAPbCl}_{1.50} \mathrm{Br}_{1.50}$ substrates with thin film for compressive strain conditions (sub-100 nm, $-2.4 \%$ strained; pink) and thick film for strainfree conditions (approximately 10 $\mathrm{mm}$, strain-free; black). The films are remeasured after 1 day or 360 days to confirm the perovskite phase stability. In the XRD and PL spectra, $a, \delta$, and $\mathrm{S}$ indicate $a-\left.F A P b\right|_{3}, \delta-\mathrm{FAPb}_{3}$, and substrate, respectively. The inset figures in (b) show photographs of two samples (black for a compressively strained film and yellow for a strain-free film). In the Raman spectra, peaks at 108 and $143 \mathrm{~cm}^{-1}$ indicate $\delta$ - and a-FAPbl ${ }_{3 \prime}$ respectively. $\mathbf{d}$ Schematic illustration of the experimental setup applying different strains and resulting photographs of the corresponding MAPbl 3 films after 500 h of illumination. e Out-of-plane XRD patterns of the $\mathrm{MAPbl}_{3}$ films with different strains by bending, as shown in (d). $\mathbf{f}$ Activation energy of ion migration as a function of the strain level in the $\mathrm{MAPb}_{3}$ films. Reprinted with permission from Ref. ${ }^{25}$. Copyright 2017 American Association for the Advancement of Science and Ref. ${ }^{32}$. Copyright 2020 Nature Publishing Group.

(Fig. 8c). The compressive strain is induced by using a lattice-mismatched substrate with a lower lattice parameter (please see section "Heteroepitaxial interface". Heteroepitaxial interface for details), while the strain-free property is simply acquired by increasing the film thickness above the critical thickness for elastic relaxation. The critical thickness of the perovskite film for elastic relaxation is approximately $3 \mu \mathrm{m}$. Therefore, the $10 \mu \mathrm{m}$-thick film is suitable to be used as a strain-free film through elastic relaxation. In Fig. 8a, the XRD patterns of the $\alpha$ phase, $<001>$ and $<002>$, consistently remain even after $360 \mathrm{~d}$ under $-2.4 \%$ (compressive) residual strain. On the other hand, the strain-free $\alpha$ phase immediately turns into the $\delta$ phase only after one day. The outstanding phase stability of $\alpha-\mathrm{FAPbI}_{3}$ with compressive strain is confirmed by the PL and Raman results. The stable $\alpha$ phase under compressive strain can be understood in terms of the removal of the driving force for the phase transition. The residual tensile strain in $\alpha-\mathrm{FAPbI}_{3}$, inherited from the film annealing process ${ }^{32}$, acts as a driving force and thus strongly drives the $\alpha$ phase to transform into the $\delta$ phase $^{48}$. The compressive strain nullifies the driving force and inhibits the structural deformation for the phase transition from occurring ${ }^{32,48}$. Furthermore, the lower interface energy between the same $\alpha$ phases may also contribute to phase stabilization to some extent. The interface energy between the cubic substrate and the cubic $\alpha$ phase is lower than that with the $\delta$ phase, energetically favoring the phase. Interestingly, the improved stability of the $\mathrm{MAPbI}_{3}$ film is also observed under strain in a certain direction ${ }^{25}$. Figure $8 \mathrm{~d}$ shows schematic illustrations of the strained $\mathrm{MAPbI}_{3}$ films in different ways by using bendable substrates. The strained films on the convex, flat, and concave substrates are exposed to $\sim 50 \mathrm{~mW} / \mathrm{cm}^{2}$ at $65{ }^{\circ} \mathrm{C}$ with encapsulation. After $500 \mathrm{~h}$ of stress, the strained film on concave substrates demonstrates outstanding phase stability, while decomposition into $\mathrm{PbI}_{2}$ is observed for the flat film as well as (to a more pronounced extent) for the convex film (Fig. 8e). The underlying mechanism is closely correlated to the ion migration energy $\left(E_{\mathrm{a}}\right)$. The $E_{\mathrm{a}}$ values of the convex, flat, and concave $\mathrm{MAPbI}_{3}$ films are $0.29,0.39$, and $0.53 \mathrm{eV}$, respectively, in the dark and 0.046, 0.074, and $0.083 \mathrm{eV}$, respectively, under $25 \mathrm{~mW} / \mathrm{cm}^{2}$ illumination, as plotted in Fig. 8f. Therefore, the reduced $E_{\mathrm{a}}$ of the convex $\mathrm{MAPbI}_{3}$ film accelerates ion migration, facilitating decomposition as well as strain relaxation, whereas the increased $E_{\mathrm{a}}$ of the concave $\mathrm{MAPbI}_{3}$ film effectively suppresses ion migration. 
Indeed, the strain-involved $\mathrm{MAPbI}_{3}$ stability behavior is closely in line with the strain effect on $\alpha-\mathrm{FAPbI}_{3}$ phase stability from the perspective of the strain tendency itself. The bending process for the $\mathrm{MAPbI}_{3}$ film varies the strain, particularly along the in-plane direction. The perovskite film typically has a tensile strain, emerging from the annealing process followed by cooling (see "Annealing process" for discussion $)^{25,32,35}$. The compressive strain applied by concave bending hence effectively cancels the residual tensile strain in the flat $\mathrm{MAPbI}_{3}$ film, while convex bending aggravates the existing strain by intensifying the tensile strain. In conclusion, the in-plane compressive strain is beneficial for the phase stability of the overall halide perovskite films.

Notably, the tensile strain formed at the interface enables the black $\gamma$-phase of $\mathrm{CsPb}_{3}$ to be energetically preferred over the yellow $\delta$-phase at room temperature ${ }^{49}$. When $\alpha$ $\mathrm{CsPb}_{3}$, formed at $300{ }^{\circ} \mathrm{C}$, is cooled to room temperature, free-standing isotropic $\alpha-\mathrm{CsPb}_{3}$ crystals turn into the photoinactive $\delta$-phase because the unstrained yellow $\delta$ phase is thermodynamically more stable than the $\gamma$-phase, showing $E_{\gamma}-E_{\delta}=85 \mathrm{meV}$. However, a more pronounced volume reduction is required for the $\delta$-phase $\left(229 \AA^{3}\right.$ at $0 \mathrm{~K}$ ) than for the $\gamma$-phase $\left(241 \AA^{3}\right.$ at $\left.0 \mathrm{~K}\right)$, which introduces different energy penalties for the strained films. The anisotropic (in-plane tensile) strain effectively hinders the black $\gamma-\mathrm{CsPb}_{3}$ from converting to the $\delta$-phase, ensuring the phase stability of the black $\mathrm{CsPbI}_{3}{ }^{49}$.

\section{Affecting parameters}

The residual strain existing in the perovskite film is significantly determined by experimental conditions, including the perovskite material composition ${ }^{35}$, a substrate for film growth ${ }^{32}$, and external stimuli ${ }^{50-52}$ during the process and the measurement. In this regard, various affecting parameters during the experiments are discussed in the following paragraphs to understand their effect on the crystal and establish a strategy for deriving the most beneficial optoelectronic properties from halide perovskite films by tailoring the strain.

\section{Annealing process (thermal stress)}

As shown in Fig. 9a, various forms of $\mathrm{MAPbI}_{3}$ perovskite crystals are prepared based on different methods to modulate the lattice strain and subsequently studied by XRD. It is apparent that the annealed perovskite film (indicated as AF), prepared by a routine annealing process at $100^{\circ} \mathrm{C}$, exhibits a strain, leading to a slight XRD peak shift compared to the case of the single-crystal powder (SCP), which is a standard control sample for the strainfree crystal. The residual strain is formed by the annealing process because the nonannealed film (NAF) shows peak positions consistent with those of the single-crystal powder (SCP). The strain in the annealed film is readily removable by scraping the film to a powder form (SP), implying that the residual strain is maintained by adhesion to the substrate. It is notable that the XRD patterns of films mostly represent out-of-plane diffraction, as shown in Fig. 9c, which mainly reflects the plane distance parallel to the substrate. On the other hand, the in-plane information can be obtained as illustrated in Fig. 9d. A strain in a certain direction usually brings about an opposite strain component in the other direction for overall relaxation ${ }^{25}$. The tensile (compressive) in-plane (out-of-plane) strain induces a minor compressive (tensile) out-of-plane (in-plane) strain or vice versa. Therefore, the increased in-plane lattice distance $\left(d_{\perp}\right)$, indicative of tensile strain, is accompanied by the reduced out-ofplane lattice distance $\left(d_{\|}\right)$, indicating compressive strain, as evidenced by the opposite XRD peak shift feature in Fig. 9b.

A gradual peak shift to a higher $2 \theta$ angle is observed from the free-standing $\mathrm{MAPbI}_{3}$ crystals during the cooling process from $100^{\circ} \mathrm{C}$ to $25^{\circ} \mathrm{C}$, owing to lattice shrinkage (Fig. 10a, c). Remarkably, the out-of-plane lattice contraction during cooling is more prominent in the perovskite film (Fig. 10b, d), which is accordingly ascribed to the lattice strain induced by film adhesion to the substrate. The shrinkage in the out-of-plane interplanar distance is more pronounced, resulting in a significant $\Delta d_{\|}$, to compensate for the restrained in-plane contraction, leading to substantial in-plane tensile strain with negligible $\Delta d_{\perp}$. In other words, the induced strain in the perovskite film is greatly governed by a difference in the thermal expansion coefficient $(\alpha)$ between the perovskite and the substrate. The $\alpha$ of $\mathrm{MAPbI}_{3}$ is $6.1 \times 10^{-5} \sim 1.57 \times$ $10^{-4} \mathrm{~K}^{-1} 25,53$, which is approximately one order of magnitude higher than that of common substrates (e.g., $\left.\alpha_{\text {glass }}=3.7 \times 10^{-6} \mathrm{~K}^{-1}, \quad \alpha_{\text {ITO }}=8.5 \times 10^{-6} \mathrm{~K}^{-1}\right)^{54} . \quad$ The perovskite film crystallized at high temperature should be cooled down to room temperature. Consequently, it is reasonable to expect the residual tensile strain along the in-plane direction of the annealed perovskite. The greater strain is induced not only by a larger difference in $\alpha_{T}$ between the perovskite and the substrate but also by a higher temperature used for perovskite annealing, regardless of the perovskite composition $\left(\mathrm{MAPbI}_{3}, \alpha-\right.$ $\mathrm{FAPbI}_{3}$, or any mixed composition) and the fabrication method (one-step, two-step, or antisolvent) ${ }^{25}$. Otherwise, a low-temperature-based process for perovskite crystallization can be another option to form a perovskite film with negligible strain dominated by the interface.

\section{Heteroepitaxial interface}

The residual tensile strain induced during the cooling process after annealing is unfavorable, especially for the phase stability of the perovskite film. Furthermore, the optoelectronic properties of the perovskite film are 

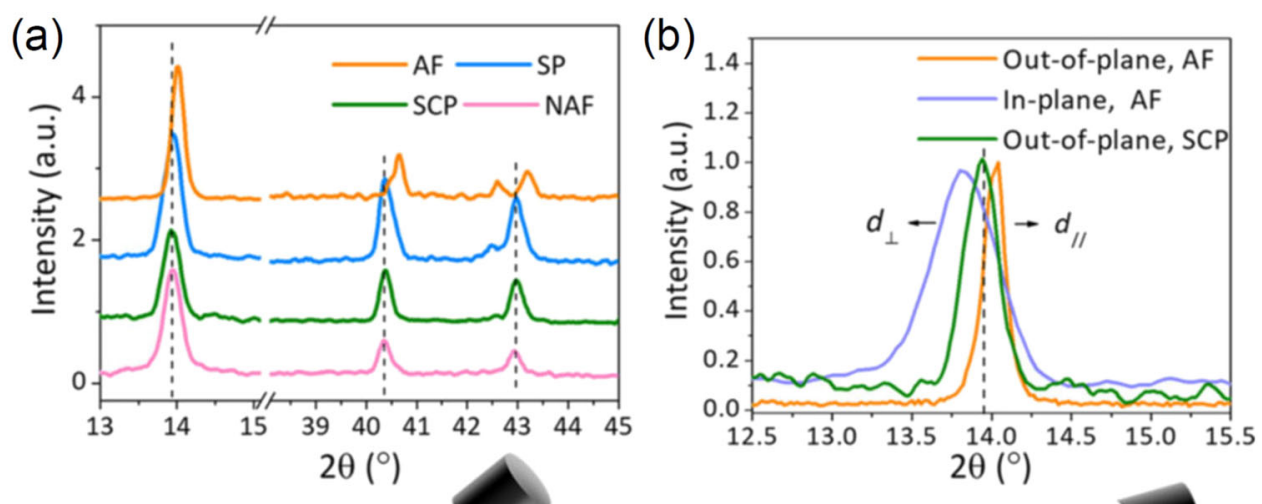

(c)

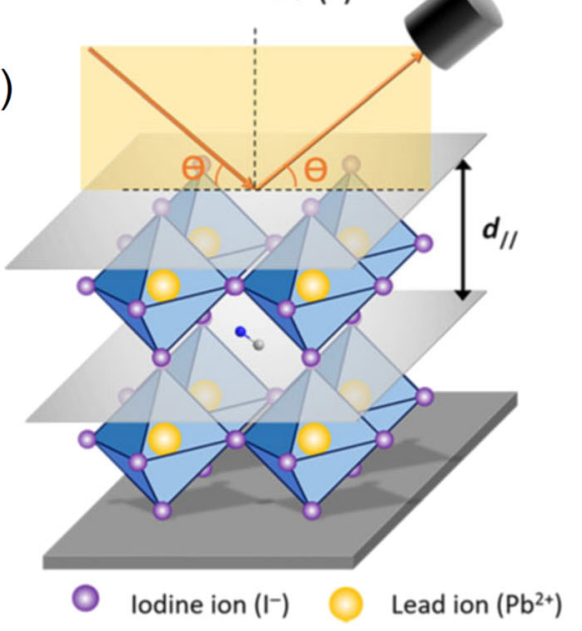

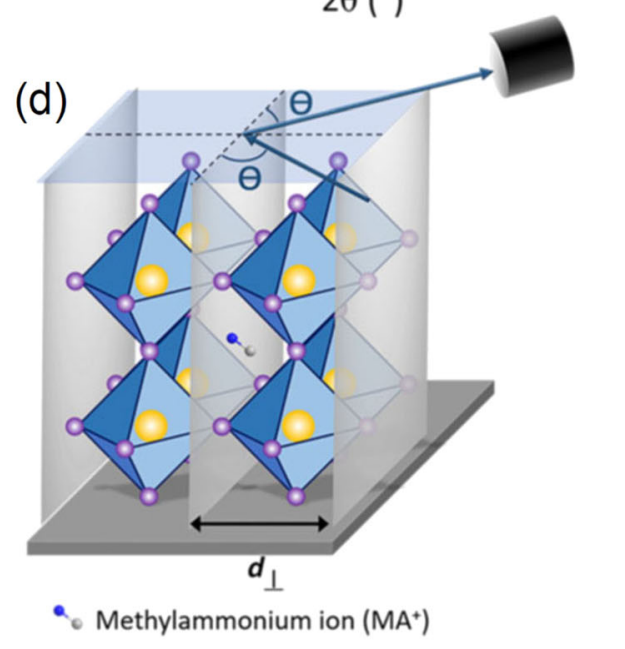

Fig. 9 Effect of annealed film on in-plane and out-of-plane lattice distance. a Out-of-plane XRD patterns of MAPbl ${ }_{3} \mathrm{Crystals}_{\mathrm{in}}$ the form of annealed film (AF), scraped powder (SP), single-crystal powder (SCP), and nonannealed film (NAF). b In-plane and out-of-plane XRD patterns of the AF and out-of-plane XRD pattern of SCP as a reference. Schematic illustrations of the (c) out-of-plane and (d) in-plane XRD measurement setup. Reprinted with permission from Ref. ${ }^{25}$. Copyright 2017 American Association for the Advancement of Science.

tunable in a sophisticated manner by modulating its internal strain. To take advantage of strain engineering for perovskite-based electronics, the induced strain should remain at the device operating conditions, usually room temperature and ambient pressure. In this regard, tuning the strain of the perovskite is attempted by the lattice mismatch at the interface with a substrate, which has a significant impact on the crystal growth of the epilayer (the perovskite film) ${ }^{32}$. Single-crystalline $\mathrm{MAPbCl}_{x} \mathrm{Br}_{3-x}$ crystals with cubic phase, synthesized by the inverse temperature growth method ${ }^{55}$, are used as substrates for $\alpha-\mathrm{FAPbI}_{3}$. The lattice parameter of the single-crystalline substrate $\left(d_{\text {sub }}\right)$ is controlled by the ratio between $\mathrm{Cl}$ and $\mathrm{Br}$ in $\mathrm{MAPbCl}_{\mathrm{x}} \mathrm{Br}_{3-x}$, where the increase in $\mathrm{x}$ results in the XRD peak shifting toward a higher $2 \theta$ angle, indicating a reduction in the lattice parameter of the substrate (Fig. 11a). The $\alpha-\mathrm{FAPbI}_{3}$ film grown on the singlecrystalline substrate shows a gradual peak shift as a function of $d_{\text {sub }}$. The lattice mismatch between $\alpha-\mathrm{FAPbI}_{3}$ and the substrate is increased by decreasing $d_{\text {sub }}$ based on lattice parameters of $6.35 \AA$ for strain-free $\alpha-\mathrm{FAPbI}_{3}$ and
5.83-5.95 $\AA$ for $\mathrm{MAPbCl}_{x} \mathrm{Br}_{3-x}{ }^{32}$. The increase in lattice mismatch leads to a $\alpha-\mathrm{FAPbI}_{3}(001)$ peak shift to a lower $2 \theta$ angle (Fig. 11a), implying an increased lattice parameter along the out-of-phase direction as the in-plane compressive strain is applied by the lattice mismatch. The lattice strain level of $\alpha-\mathrm{FAPbI}_{3}$ ranging from $-0.8 \%$ to $-2.4 \%$ is determined by the compositional variation of $\mathrm{MAPbCl}_{\mathrm{x}} \mathrm{Br}_{3-\mathrm{x}}(0 \leq x \leq 1.5)$. $Q_{\mathrm{x}}$ and $Q_{z}$ shown in Fig. 11b correspond to the in-plane and out-of-plane reciprocal space coordinates, respectively. The reciprocal space mapping shows a decreased in-plane lattice parameter as well as an increased out-of-plane lattice parameter under the high strain level. A pronounced tetragonality of $\alpha$ $\mathrm{FAPbI}_{3}$ is therefore estimated by increasing the lattice mismatch from $0 \%$ (strain-free) to $-1.2 \%$ and $-2.4 \%$ due to lattice deformation by epitaxial compressive strain. Thus, epitaxial crystal growth for strain-engineered perovskite films seems very effective and intuitive. The limit of this approach is the difficulty in acquiring a feasible highly crystalline substrate with the required lattice parameter for perovskite-based electronics. 

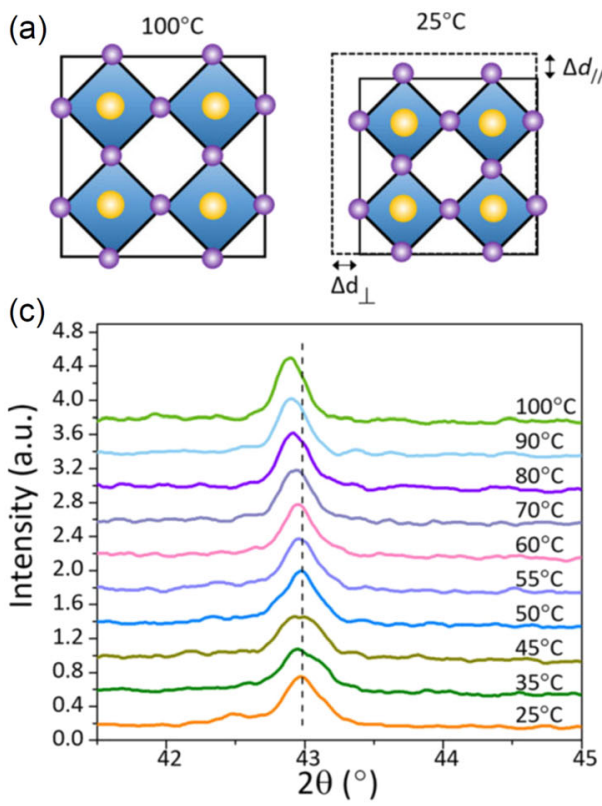

(b)
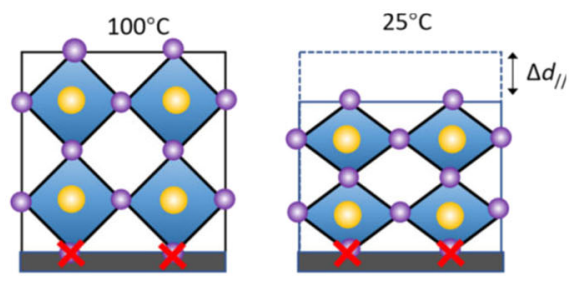

(d)

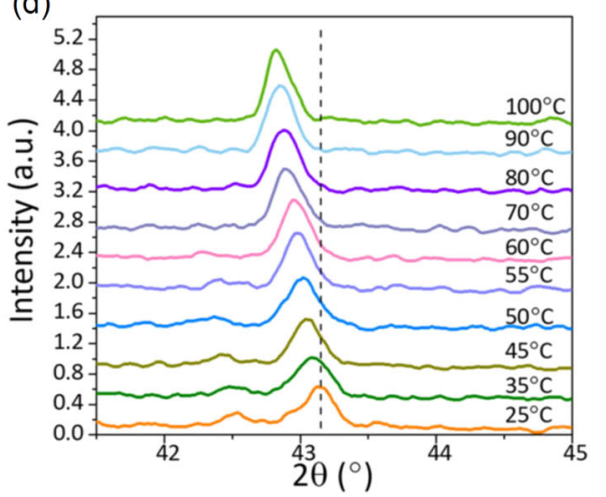

Fig. 10 Effect of temperature on out-of-plane interplanar distance. Schematic illustration showing the temperature effect on the perovskite structure (a) in the form of scraped powder and (b) as a film in the presence of substrate. In situ out-of-plane XRD patterns of (c) the scraped powder and (d) the film at different temperatures. Reprinted with permission from Ref. ${ }^{25}$. Copyright 2017 American Association for the Advancement of Science.

(a)

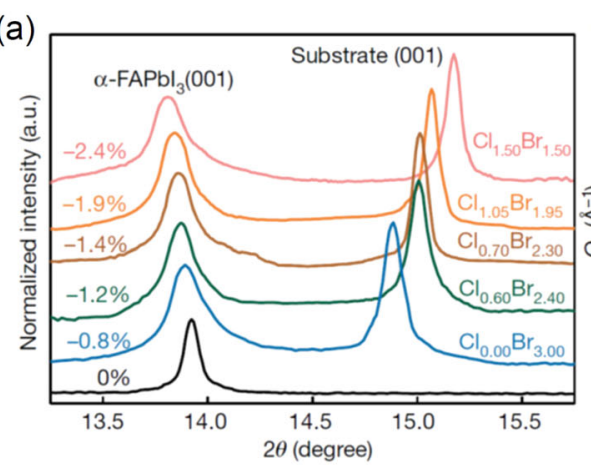

(b)

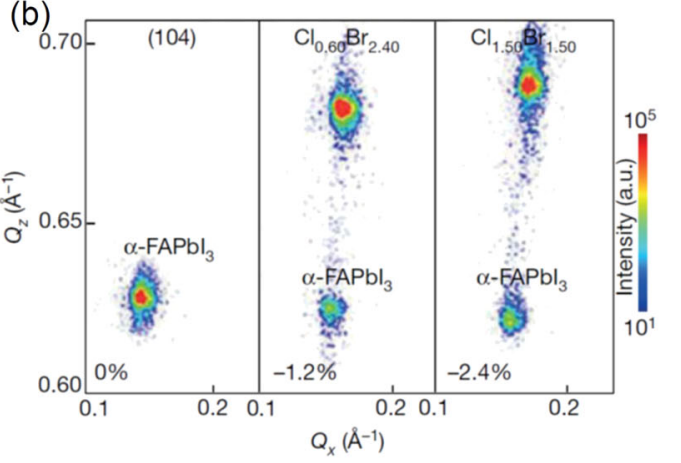

Fig. 11 Control of residual strain by lattice mismatch between perovskite film and substrate. a High-resolution XRD $\omega-2 \theta$ scan of the (001) peaks of a-FAPbl 3 films grown on different substrates to modulate lattice mismatch. $\mathbf{b}$ Reciprocal space maps with (104) asymmetric reflection of the a-FAPbl ${ }_{3}$ films depending on the lattice mismatch with the substrate. $Q_{x}$ and $Q_{z}$ are the in-plane and out-of-plane reciprocal space coordinates, respectively. Reprinted with permission from Ref. ${ }^{32}$. Copyright 2020 Nature Publishing Group.

\section{Compositional material inhomogeneity}

The chemical pressure induced by employing foreign ions is represented as lattice strain in crystals ${ }^{56,57}$. The inhomogeneity in a mixed perovskite film occasionally leads to a vertical gradient in residual strain ${ }^{35}$. The crystal structure of the $\left(\mathrm{FAPbI}_{3}\right)_{0.85}\left(\mathrm{MAPbBr}_{3}\right)_{0.15}$ perovskite film is investigated by measuring depth-dependent nanobeam electron diffraction patterns. Depths of $50 \mathrm{~nm}$ (b), $200 \mathrm{~nm}$ (c), and $500 \mathrm{~nm}$ (d) from the top surface are chosen for the probe, as indicated in Fig. 12a. The nanobeam electron diffraction patterns reveal that the lattice distance, $d$, is increased from $1.60 \AA$ to $1.64 \AA$ and $1.67 \AA$ as the probe spot varies from $50 \mathrm{~nm}$ to $100 \mathrm{~nm}$ and $500 \mathrm{~nm}$ deep, respectively (Fig. 12b-d). Furthermore, the depthdependent GIXRD also confirms the microscopic crystal inhomogeneity, where a peak shift to a lower $2 \theta$, indicative of the increased $d$ along the out-of-plane direction, is monitored as the probe depth is increased. The slope and the sign of the linear plot, $2 \theta$ as a function of $\sin ^{2} \phi$, correspond to the residual strain level and its direction, respectively ${ }^{36,37}$. In Fig. 12e, the fitted lines have a negative slope regardless of the probe depth, which indicates 

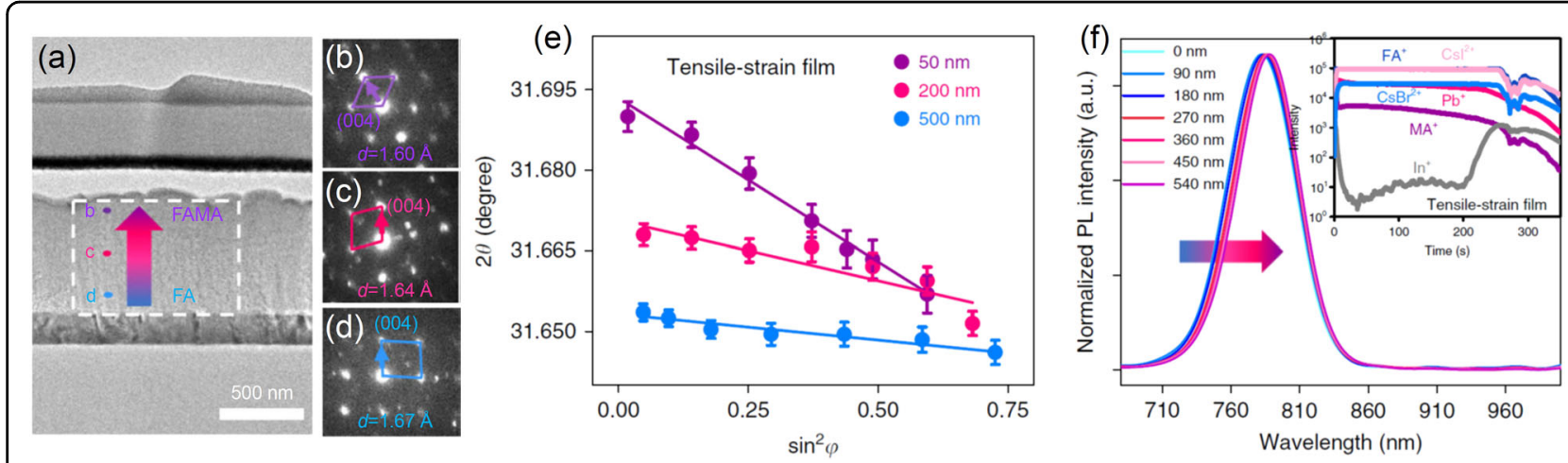

Fig. 12 Vertical gradient in residual strain due to depth-dependent compositional difference in perovskite film. a Cross-sectional TEM images of a $\left(\mathrm{FAPb}_{3}\right)_{0.85}\left(\mathrm{MAPbBr}_{3}\right)_{0.15}$ film, where the spots marked as $\mathrm{b}, \mathrm{c}$, and $\mathrm{d}$ are used for the diffraction patterns in (b), (c), and (d) for depth profiling, respectively. $\mathbf{b}$ - $\mathbf{d}$ Nanobeam electron diffraction patterns of (004) corresponding to the marked spots in (a). e The $2 \theta$ - $\sin ^{2} \varphi$ plots to assume residual strain at depths of 50,200, and 500 nm from the surface for the ( $\left.\mathrm{FAPbl}_{3}\right)_{0.85}\left(\mathrm{MAPbBr}_{3}\right)_{0.15}$ film under tensile strain. The error bar indicates the standard deviation of $2 \theta$. $\mathbf{f} \mathrm{PL}$ confocal fluorescence microscopy of the ( $\left(\mathrm{FAPb}_{3}\right)_{0.85}\left(\mathrm{MAPbBr}_{3}\right)_{0.15}$ film at depths from Onm (top surface) to $540 \mathrm{~nm}$ (near the bottom surface with substrate). The inset figure in (f) shows the TOF-SIMS depth profiles of the sample. Reprinted with permission from Ref. ${ }^{35}$. Copyright 2019 Nature Publishing Group.

that $d$ starts increasing by reflecting the in-plane strain component, corresponding to an in-plane tensile strain across the entire film. On the other hand, the strain level is highly governed by the probe depth, showing a higher absolute value of the slope near the top surface $(50 \mathrm{~nm}$ deep) and a lower absolute value of the slope near the substrate $(500 \mathrm{~nm}$ deep). The gradient in the in-plane tensile strain emerges vertically in the $\left(\mathrm{FAPbI}_{3}\right)_{0.85}$ $\left(\mathrm{MAPbBr}_{3}\right)_{0.15}$ film, where the intense strain at the top surface is gradually relaxed with increasing depth to the bottom substrate. The observed gradient in the residual strain is well matched with the PL depth profile in Fig. 12f, where a gradual PL peak shift reflects the gradually decreasing tensile strain from the top to the bottom, leading to a lower $V_{\mathrm{g}}$ toward the bottom due to the increased antibonding overlap by relaxing the tensile strain. It is quite unusual for the in-plane tensile strain to be more intense at the top than at the bottom. The induced tensile strain usually prevails at the interface adjacent to the substrate because the tensile strain is highly governed by the $\alpha$ difference between the perovskite and the substrate, which typically results in the opposite trend ${ }^{25,32}$. This distinctive strain gradient, particularly in the mixed perovskite film, seems to originate from the evolution of the different perovskite compositions depending on the depth, as evidenced by the timeof-flight secondary ion mass spectrometry (TOF-SIMS) results shown in the inset of Fig. 12f. These results show an inconsistent depth profile of $\mathrm{MA}^{+}$with an $\mathrm{MA}^{+}$-deficient region near the bottom substrate, in accordance with the decreased $d$ along the out-of-plane direction near the top surface by employing a higher concentration of $\mathrm{MA}^{+}$. Therefore, the estimation of the residual strain should be carried out with care, especially in mixed perovskites, after considering the compositional inhomogeneity and distribution.

\section{Summary and perspective}

Halide perovskite films based on polycrystalline crystals exhibit strain inhomogeneity, which readily interferes with the bonding strength and thus changes the optoelectronic properties as well as the phase stability. The tensile strain along the in-plane direction arises in the perovskite film during cooling after the film is annealed. The residual tensile strain is mostly responsible for the unfavorable carrier dynamics and the phase stability as well. The overall characteristics of the perovskite crystals are improved by relieving the internal in-plane tensile strain and even further enhanced by inducing the residual compressive strain along the in-plane direction. Inability to control the residual strain would be a great barrier for perovskite-based electronics, whereas strain engineering is a powerful strategy for a sophisticatedly tailored lattice structure to derive demanding properties. Therefore, it is highly necessary to develop a strain tailoring method that is practically compatible with the process conditions for real devices where the intended residual strain remains intact under the device operating conditions. A lowtemperature process, epilayer growth on a pre-expanded substrate, or lattice-mismatched substrate compatible with carrier extraction can be an option to fully control the residual strain and thus tune the optoelectronic properties with the ensured stability.

\section{Acknowledgements}

This work was supported by National Research Foundation of Korea (NRF) grants funded by the Ministry of Science, ICT Future Planning (MSIP) of Korea under contracts NRF-2012M3A6A7054861 (Global Frontier R\&D Program on Center for Multiscale Energy System), NRF-2016M3D1A1027663, NRF- 
2016M3D1A1027664 (Future Materials Discovery Program), NRF2015M1A2A2053004 (Climate Change Management Program) and NRF2020R1F1A1075558.

\section{Conflict of interest}

The authors declare that they have no conflict of interest.

\section{Publisher's note}

Springer Nature remains neutral with regard to jurisdictional claims in published maps and institutional affiliations.

Received: 13 March 2020 Revised: 14 September 2020 Accepted: 18 September 2020.

Published online: 11 December 2020

\section{References}

1. Kim, H.S. et al. Lead iodide perovskite sensitized all-solid-state submicron thin film mesoscopic solar cell with efficiency exceeding 9\%. Sci. Rep. 2, 591 (2012).

2. Lee, M. M., Teuscher, J., Miyasaka, T., Murakami, T. N. \& Sanith, H. J. Efficient hybrid solar cells based on meso-superstructured organometal halide perovskites. Science 338, 643-647 (2012).

3. Best Research-Cell Efficiencies Chart. https:/www.nrel.gov/pv/assets/pdfs/ best-research-cell-efficiencies.20200925.pdf. Accessed 12 Nov 2020.

4. Park, N.-G., Zhu, K. Scalable fabrication and coating methods for perovskite solar cells and solar modules. Nat. Rev. Mater. https:/doi.org/10.1038/s41578019-0176-2 (2020).

5. Lee, J.-W. Lee, D.-K, Jeong, D.-N. \& Park, N.-G. Control of crystal growth toward scalable fabrication of perovskite solar cells. Adv. Funct. Mater. 29, 1807047 (2018).

6. Huang, F., Li, M., Siffalovic, P., Cao, G. \& Tian, J. From scalable solution fabrication of perovskite films towards commercialization of solar cells. Energy Environ. Sci. 12, 518-549 (2019).

7. Kim, H. S., Hagfeldt, A. \& Park, N.-G. Morphological and compositional progress in halide perovskite solar cells. Chem. Commun. 55, 1192-1200 (2019).

8. Turren-Cruz, S.H., Hagfeldt, A. \& Saliba, M. Methylammonium-free, highperformance and stable perovskite solar cells on a planar architecture. Science 362, 449-453 (2018).

9. Seo, J.-Y. et al. Novel p-dopant toward highly efficient and stable perovskite solar cells. Energy Environ. Sci. 11, 2985-2992 (2018).

10. Yang, T.-Y. et al. Achieving long-term operational stability of perovskite solar cells with a stabilized efficiency exceeding $20 \%$ after 1000 h. Adv. Sci. 6 , 1900528 (2019).

11. Jung, E. H. et al. Efficient, stable and scalable perovskite solar cells using poly(3hexylthiophene). Nature 567, 511-515 (2019).

12. Xiao, M. et al. A fast deposition-crystallization procedure for highly efficient leas iodide perovskite thin-film solar cells. Angew. Chem. Int. Ed. 53, 9898-9903 (2014).

13. Ahn, N. et al. Highly reproducible perovskite solar cells with average efficiency of $18.3 \%$ and best efficiency of $19.7 \%$ fabricated via lewis base adduct of lead (II) iodide. J. Am. Chem. Soc. 137, 8696-8699 (2015).

14. Yin, W.-J., Shi, T. \& Yan, Y. Unique properties of halide perovskites as possible origins of the superior solar cell performance. Adv. Mater. 26, 4653-4658 (2014).

15. D'Innocenzo, V. et al. Excitons versus free charges in organo-lead tri-halide perovskites. Nat. Commun. 5, 3586 (2014).

16. Giorgi, G., Fujisawa, J.-I., Segawa, H. \& Yamashita, K. Small photocarrier effective masses featuring ambipolar transport in methylammonium lead iodide perovskite: a density functional analysis. J. Phys. Chem. Lett. 4, 4213-4216 (2013).

17. Stranks, S. D. et al. Electron-hole diffusion lengths exceeding 1 micrometer in an organometal trihalide perovskite absorber. Science 342, 341-344 (2013).

18. Liu, Y. et al. Ultrahydrophobic 3D/2D fluoroarene bilayer-based water-resistant perovskite solar cells with efficiencies exceeding 22\%. Sci. Adv. 5, eaaw2543 (2019).

19. Li, M.-H. et al. Highly efficient 2D/3D hybrid perovskite solar cells via lowpressure vapor-assisted solution process. Adv. Mater. 30, e1801401 (2018).

20. Grancini, G. et al. One-year stable perovskite solar cells by 2D/3D interface engineering. Nat. Commun. 8, 15684 (2017).

21. Kurdi, M. E. et al. Control of direct band gap emission of bulk germanium by mechanical tensile strain. Appl. Phys. Lett. 96, 041909 (2010).
22. Ahn, G. H. et al. Strain-engineered growth of two-dimensional materials. Nat. Commun. 8, 608 (2017)

23. Hubbard, S. M. et al. Effect of strain compensation on quantum dot enhanced GaAs solar cells. Appl. Phys. Lett. 92, 123512 (2008).

24. Bush, K. A. et al. Controlling thin-film stress and wrinkling during perovskite film formation. ACS Energy Lett. 3, 1225-1232 (2018).

25. Zhao, J. et al. Strained hybrid perovskite thin films and their impact on the intrinsic stability of perovskite solar cells. Sci. Adv. 3, eaao5616 (2017).

26. Meloni, S., Palermo, G., Ashari-Astani, N., Grätzel, M. \& Rothlisberger, U. Valence and conduction band tuning in halide perovskites for solar cell applications. J. Mater. Chem. A 4, 15997-16002 (2016).

27. Yin, W. J. et al. Halide perovskite materials for solar cells: a theoretical review. J. Mater. Chem. A 3, 8926-8942 (2015).

28. Zheng, $X$. et al. Improved phase sstability of formamidinium lead triiodide perovskite by strain relaxation. ACS Energy Lett. 1, 1014-1020 (2016).

29. Leblebici, S. Y. et al. Facet-dependent photovoltaic efficiency variations in single grains of hybrid halide perovskite. Nat. Energy 1, 16093 (2016).

30. Jones, T. W. et al. Lattice strain causes non-radiative losses in halide perovskites. Energy Environ. Sci. 12, 596-606 (2019).

31. Pohl, U. W. Epitaxy of Semiconductors: Introduction to Physical Principles (Springer, 2013).

32. Chen, Y. et al. Strain engineering and epitaxial stabilization of halide perovskites. Nature 577, 209-215 (2020).

33. Prasanna, R. et al. Band gap tuning via lattice contraction and octahedral tilting in perovskite materials for photovoltaics. J. Am. Chem. Soc. 139, 11117-11124 (2017).

34. Murali, B. et al. Temperature-induced lattice relaxation of perovskite crystal enhances optoelectronic properties and solar cell performance. J. Phys. Chem. Lett. 8, 137-143 (2017).

35. Zhu, C. Strain engineering in perovskite solar cells and its impacts on carrier dynamics. Nat. Commun. 10, 815 (2019).

36. Chen, Z., Prud'homme, N., Wang, B. \& Ji, V. Residual stress gradient analysis with GIXRD on $\mathrm{ZrO}_{2}$ thin films deposited by MOCVD. Surf. Coat. Technol. 206 405-410 (2011)

37. Peng, J. et al. Residual stress gradient analysis by the GIXRD method on CVD tantalum thin films. Surf. Coat. Technol. 200, 2738-2743 (2006).

38. Boopathi, K. M. et al. Permanent lattice compression of lead- halide perovskite for persistently enhanced optoelectronic properties. ACS Energy Lett. 5, 642-649 (2020).

39. Ghimire, S., Takahashi, K., Takano, Y., Nakamura, T. \& Biju, V. Photon recycling by energy transfer in piezochemically synthesized and close-packed methylammonium lead halide perovskites. J. Phys. Chem. C. 123, 27752-27758 (2019).

40. Cao, Y. et al. Pressure-tailored band gap engineering and structure evolution of cubic cesium lead iodide perovskite nanocrystals. J. Phys. Chem. C. 122 9332-9338 (2018).

41. Wang, Y. et al. Pressure-induced phase transformation, reversible amorphization, and anomalous visible light response in organolead bromide perovskite. J. Am. Chem. Soc. 137, 11144-11149 (2015).

42. Blakesley, J. C. et al. Towards reliable charge-mobility benchmark measurements for organic semiconductors. Org. Electron. 15, 1263-1272 (2014).

43. Sherkar, T. et al. Recombination in perovskite solar cells: significance of grain boundaries, interface traps, and defect ions. Acs Energy Lett. 2, 1214-1222 (2017).

44. deQuilettes, D. W. et al. Impact of microstructure on local carrier lifetime in perovskite solar cells. Science 348, 683-686 (2015).

45. Stranks, S. D. et al. Recombination kinetics in organic-inorganic perovskites: excitons, free charge, and subgap states. Phys. Rev. Appl. 2, 034007 (2014).

46. Han, Q. et al. Single crystal formamidinium lead iodide ( $\left(\mathrm{FAPb}_{3}\right)$ : insight into the structural; optical, and electrical properties. Adv. Mater. 28, 2253-2258 (2016).

47. Xie, L-Q. et al. Understanding the cubic phase stabilization and crystallization kinetics in mixed cations and halides perovskite single crystals. J. Am. Chem. Soc. 139, 3320-3323 (2017).

48. Saidaminov, M. I. et al. Suppression of atomic vacancies via incorporation of isovalent small ions to increase the stability of halide perovskite solar cells in ambient air. Nat. Energy 3, 648-654 (2018).

49. Steele, J. A. et al. Thermal unequilibrium of strained black $\mathrm{CsPb}_{3}$ thin films Science 365, 679-684 (2019).

50. Liu, G. et al. Pressure-induced bandgap optimization in lead-based perovskites with prolonged carrier lifetime and ambient retainability. Adv. Funct. Mater. 27 1604208 (2017). 
51. Chen, B. et al. Large electrostrictive response in lead halide perovskites. Nat. Mater. 17, 1164 (2018).

52. Wang, Y. et al. Nontrivial strength of van der Waals epitaxial interaction in soft perovskites. Phys. Rev. Mater. 2, 076002 (2018).

53. Jacobsson, T. J., Schwan, L. J., Ottosson, M., Hagfeldt, A. \& Edvinsson, T. Determination of thermal expansion coefficients and locating the temperature-induced phase transition in methylammonium lead perovskites using X-ray diffraction. Inorg. Chem. 54, 10678-10685 (2015).

54. Craciun, V., Craciun, D., Wang, X., Anderson, T. J. \& Singh, R. K. Transparent and conducting indium tin oxide thin films grown by pulsed laser deposition at low temperatures. J. Optoelectron. Adv. Mater. 25 401-408 (2003).

55. Maculan, $\mathrm{G}$. et al. $\mathrm{CH}_{3} \mathrm{NH}_{3} \mathrm{PbCl}_{3}$ single crystals: inverse temperature crystallization and visible-blind UV-photodetector. J. Phys. Chem. Lett. 6, 3781-3786 (2015).

56. Ghosh, D., Smith, A. R., Walker, A. B., Islam, M. S. \& Mixed, A. Cation perovskites for solar cells: atomic-scale insights into structural distortion, hydrogen bonding, and electronic properties. Chem. Mater. 30, 5194-5204 (2018).

57. Ghosh, D., Aziz, A., Dawson, J. A., Walker, A. B. \& Islam, M. S. Putting the squeeze on lead iodide perovskites: pressure-induced effects to tune their structural and optoelectronic behavior. Chem. Mater. 31, 4063-4071 (2019). 